\title{
Ergodic approximations via matrix regularization approach
}

\author{
Giuseppe Marino ${ }^{1,2 *}$, Filomena Cianciaruso ${ }^{1}$ and Nawab Hussain ${ }^{2}$
}

\author{
"Correspondence: \\ giuseppe.marino@unical.it \\ 'Dipartimento di Matematica, \\ Universitá della Calabria, Arcavacata \\ di Rende, CS 87036, Italy \\ 2 Department of Mathematics, King \\ Abdulaziz University, P.O. Box 80203 \\ Jeddah, 21589, Saudi Arabia
}

\begin{abstract}
In this paper we use a matrix approach to approximate solutions of variational inequalities in Hilbert spaces. The methods studied combine new or well-known iterative methods (as the original Mann method) with regularized processes that involve regular matrices in the sense of Toeplitz. We obtain ergodic type results and convergence.
\end{abstract}

MSC: 47J20; 47J25; 49J40; 65J15

Keywords: monotone variational inequality; nonspreading mappings; iterative method; Cesaro means; Toeplitz matrix

\section{Introduction and results}

The results presented here will be proved in Section 2 .

Let $H$ be a Hilbert space and let $f: H \rightarrow H$ be a $\rho$-contraction. It is well known that if $T: H \rightarrow H$ is a quasi-nonexpansive mapping, the set of the fixed points $\operatorname{Fix}(T)$ is closed and convex and the variational inequality problem (VIP)

$$
\left\langle(I-f) x^{*}, y-x^{*}\right\rangle \geq 0, \quad \forall y \in \operatorname{Fix}(T)
$$

admits a unique solution.

The interactions between fixed point theory and the calculus of variations are certainly not recent. In this direction Moudafi in 2000 [1], Xu in 2004 [2] and Marino and Xu [3] in 2006 have shown deep connections between the approximation of fixed points for nonlinear mappings and the approximation of solutions of variational inequalities.

In this paper our aim is to approximate on $\operatorname{Fix}(T)$ the unique solution of a (VIP) when $T$ is a nonspreading mapping, i.e.

$$
\|T x-T y\|^{2} \leq\|x-y\|^{2}+2\langle x-T x, y-T y\rangle, \quad \forall x, y \in H
$$

The class of nonspreading mappings has been introduced recently by Kohsaka and Takahashi in [4]. They observed that it contains the class of firmly nonexpansive-type mappings, introduced by the same authors in [5]

Note that a nonspreading mapping $T$ is quasi-nonexpansive [6].

The choice to consider quasi-nonexpansive mappings is made since these operators are widely used in the sub-gradient projection operator techniques to solve convex con-

(c) 2015 Marino et al. This article is distributed under the terms of the Creative Commons Attribution 4.0 International License (http://creativecommons.org/licenses/by/4.0/), which permits unrestricted use, distribution, and reproduction in any medium, provided you give appropriate credit to the original author(s) and the source, provide a link to the Creative Commons license, and indicate if changes were made. 
strained problems (see Maingé [7] and the references contained therein). Nevertheless, the lack of continuity of a quasi-nonexpansive mapping makes it difficult to prove asymptotical stability of iterates of $T$ (see, for example, [8]). This property is a key for the convergence analysis of sequences generated by well-known iterative methods.

To fill this gap, we employ regularizations that involve matrices $\left(b_{n, k}\right)_{n, k \in \mathbb{N}}$, so-called triangular-Toeplitz matrices, satisfying the following properties:

(h0) $b_{n, k} \geq 0$ for all $n, k \in \mathbb{N}$;

(h1) for any $n \in \mathbb{N}, b_{n, k}=0$ for $k>n$ and for all $n \in \mathbb{N},\left(b_{n, k}\right)_{k \in \mathbb{N}}$ is a nonincreasing sequence;

(h2) for any $n \in \mathbb{N}, \sum_{k=1}^{n} b_{n, k}=1$, and

(h3) for any $k \in \mathbb{N}, b_{n, k} \rightarrow 0$, as $n \rightarrow \infty$.

We will denote by $\mathfrak{T}$ the set of the triangular-Toeplitz matrices; $\mathfrak{T}$ is a subclass of the regular matrices in the sense of Toeplitz (see [9]). The weak limits of a regularized sequence that involves a nonspreading mapping and a triangular-Toeplitz matrix are fixed points of $T$; more precisely, we will prove the following.

Lemma 1.1 Let $T: H \rightarrow H$ be a nonspreading mapping and let $\left(b_{n, k}\right)_{n, k \in \mathbb{N}} \in \mathfrak{T}$.

Let $\left(x_{n}\right)_{n \in \mathbb{N}}$ be a bounded sequence in $H$; we define

$$
y_{n}:=\sum_{k=0}^{n-1} b_{n, k+1} T^{k} x_{n}
$$

Then the weak limits of $\left(y_{n}\right)_{n \in \mathbb{N}}$ are fixed points of $T$, i.e. $\omega_{w}\left(y_{n}\right) \subset \operatorname{Fix}(T)$.

In 1953 Mann [10], taking in account the works of Cesaro, Fejer and Toeplitz, considered the problem to construct a sequence in a convex and compact set $C$ of a Banach space $X$ that converges to a fixed point of a continuous transformation $T: C \rightarrow C$.

Mann introduced an infinite triangular matrix $\left(a_{n, k}\right)_{n, k \in \mathbb{N}}$,

$$
\left(\begin{array}{ccccc}
1 & 0 & 0 & \ldots & 0 \\
a_{2,1} & a_{2,2} & 0 & \ldots & 0 \\
\ldots & \ldots & \ldots & \ldots & \ldots \\
a_{n, 1} & a_{n, 2} & \ldots & a_{n, n} & \ldots \\
\ldots & \ldots & \ldots & \ldots & \ldots
\end{array}\right)
$$

such that

$$
\text { (M1) } \quad a_{n, k} \geq 0, \quad \text { and } \quad a_{n, k}=0, \quad \text { for } k>n ; \quad \text { (M2) } \quad \sum_{k=1}^{n} a_{n, k}=1, \quad \forall n \text {. }
$$

We will denote by $\mathfrak{M}$ the set of matrices satisfying (M1) and (M2).

Let us observe that $\mathfrak{T} \subset \mathfrak{M}$.

By using $\left(a_{n, k}\right)_{n, k \in \mathbb{N}}$ Mann introduced the following iteration process:

$$
\left\{\begin{array}{l}
v_{n}=\sum_{k=1}^{n} a_{n, k} x_{k} \\
x_{n+1}=T\left(v_{n}\right)
\end{array}\right.
$$


It is very easy to check that the Cesaro matrix

$$
C:=\left(\begin{array}{ccccc}
1 & 0 & 0 & \ldots & 0 \\
\frac{1}{2} & \frac{1}{2} & 0 & \ldots & 0 \\
\ldots & \ldots & \ldots & \ldots & \ldots \\
\frac{1}{n} & \frac{1}{n} & \ldots & \frac{1}{n} & \ldots \\
\ldots & \ldots & \ldots & \ldots & \ldots
\end{array}\right)
$$

belongs to $\mathfrak{T}$. Moreover, by using $C$, (1.4) becomes

$$
v_{n+1}=\left(1-\frac{1}{n+1}\right) v_{n}+\frac{1}{n+1} T v_{n} .
$$

However, to the best of our knowledge, there are not also convergence results for the original Mann iterative method. Therefore, in light of the above facts, we study an iterative method that implements two matrices: $\left(a_{n, k}\right)_{n, k \in \mathbb{N}} \in \mathfrak{M}$ to compute our iterations; and $\left(b_{n, k}\right)_{n, k \in \mathbb{N}} \in \mathfrak{T}$ to regularize the mapping involved.

We will investigate on the following Mann-Toeplitz iteration process: let $x_{1} \in H$

$$
\left\{\begin{array}{l}
v_{n}=\sum_{k=1}^{n} a_{n, k} x_{k}, \\
x_{n+1}=f_{\mu_{n}} \sum_{k=0}^{n-1} b_{n, k+1} T^{k} v_{n},
\end{array}\right.
$$

where $\left(a_{n, k}\right)_{n, k \in \mathbb{N}}$ satisfies (M1) and (M2), i.e. $\left(a_{n, k}\right)_{n, k \in \mathbb{N}} \in \mathfrak{M}, T$ is a nonspreading mapping, $f$ is a $\rho$-contraction, and $f_{\mu_{n}}$ is the averaged mapping $f_{\mu_{n}}=\mu_{n} f+\left(1-\mu_{n}\right) I$ (see [11] for the regularization properties of nonspreading and averaged mappings).

The matrix $\left(b_{n, k}\right)_{n, k \in \mathbb{N}}$ in (1.6) plays a regularization role similar to the Cesaro matrix. It is well known that the Cesaro mean preserves (or it increases) the regularity of a sequence. Therefore many recent papers, concerning the approximation of fixed points of nonlinear mappings and sometimes solutions of a (VIP), combine well-known iterative methods (Mann-Dotson, viscosity, Halpern, and so on) with the Cesaro means.

The first nonlinear ergodic theorem in Hilbert space was established by Baillon in [12], who proved that, if $C$ is a nonempty closed convex subset of a Hilbert space and $T: C \rightarrow C$ is a nonexpansive mapping with $\operatorname{Fix}(T) \neq \emptyset$, the Cesaro means

$$
S_{n}(x)=\frac{1}{n} \sum_{k=0}^{n-1} T^{k}(x)
$$

weakly converges to a fixed point of $T$.

Shimizu and Takahashi in [13] studied by Cesaro means the convergence of an iteration process for a family of nonexpansive mappings in a Hilbert space. This result has been extended by Shioji and Takahashi in [14], by Song and Chen in [15] in the setting of uniformly convex Banach spaces.

However, all the cited approximation methods are not suitable for nonexpansive nonself-mappings. In 2004 Matsushita and Kuroiwa in [16] and Su and Li in 2006 (see [17]) studied two iterative methods for a nonexpansive nonself-mapping in a Hilbert space; in [18] Wangkeeree extended the results in [16] in the more general setting of uniformly convex Banach spaces. 
Many references can be cited dealing with the approximation of solutions of (VIPs) and equilibrium problems. For instance in [19, 20], and [21] the authors introduced iterative processes in Hilbert spaces by using the Cesaro means for finding a common element of the set of fixed points of a nonexpansive mapping and the set of solutions of an equilibrium problem.

In 2009 and in 2010 Song (see [22] and [23]) studied an implicit viscosity method for a continuous pseudocontractive mapping and a Mann iterations of its Cesaro mean for an asymptotically nonexpansive mapping in Banach spaces.

Nevertheless the Cesaro matrix is not the unique matrix with regularization properties. Toeplitz summability theorems proved that there exists a class of matrices including the Cesaro means with the same regularization property. Therefore we consider it relevant, in its generality, to put our attention on the class of triangular-Toeplitz matrices.

We denote by $\mathfrak{M}^{*}$ an interesting subclass of $\mathfrak{M}$ of matrices such that $a_{n, n} \rightarrow 1$ as $n \rightarrow$ $+\infty$. An example of such matrices is

$$
\tilde{A}:=\left(\begin{array}{ccccc}
1 & 0 & 0 & \ldots & 0 \\
\frac{1}{4} & \frac{3}{4} & 0 & \ldots & 0 \\
\ldots & \ldots & \ldots & \ldots & \ldots \\
\frac{1}{n^{2}(n-1)} & \frac{1}{n^{2}(n-1)} & \ldots & 1-\frac{1}{n^{2}} & \ldots \\
\ldots & \ldots & \ldots & \ldots & \ldots
\end{array}\right)
$$

In Section 2 for this subclass of matrices we will prove the following.

Theorem 1.2 Let $f: H \rightarrow H$ be a $\rho$-contraction and let $f_{\mu_{n}}$ be the averaged mapping $f_{\mu_{n}}=$ $\mu_{n} f+\left(1-\mu_{n}\right) I$. Let $T$ be a nonspreading mapping.

Let us choose $\left(a_{n, k}\right)_{k, n \in \mathbb{N}} \in \mathfrak{M}^{*}$ and $\left(b_{n, k}\right)_{n, k \in \mathbb{N}} \in \mathfrak{T}$.

Let us choose $\mu<\frac{2(1-\rho)}{(1+\rho)^{2}}$ and $\left(\mu_{n}\right)_{n \in \mathbb{N}} \subset(0, \mu)$ such that

(h4) $\lim _{n \rightarrow \infty} \mu_{n}=0, \sum_{n \in \mathbb{N}} \mu_{n}=\infty$.

(h5) $\lim _{n \rightarrow \infty} \frac{1-a_{n, n}}{\mu_{n}}=0$.

Set $\tau=1-\rho$.

Then the sequence generated by the Mann-Toeplitz iterative method (1.6) strongly converges to $x^{*} \in \operatorname{Fix}(T)$, that is, a unique solution of the variational inequality

$$
\left\langle(I-f) x^{*}, y-x^{*}\right\rangle \geq 0, \quad \forall y \in \operatorname{Fix}(T) .
$$

Remark 1.3 The assumption (h5) is easily satisfied. For example, choosing $\mu_{n}=\frac{1}{n}$ and $\tilde{A}$ as in (1.7), we see that it holds.

If $\left(\gamma_{n}\right)_{n \in \mathbb{N}}$ is a sequence in $[0,1]$, we can define, recursively, a matrix $\left(\tilde{a}_{n, k}\right)_{n, k \in \mathbb{N}}$ by

$$
\left(\tilde{a}_{n, k}\right)_{n, k \in \mathbb{N}}= \begin{cases}1, & n=k=1 \\ 0, & n \geq 1 \text { and } k>n, \\ \gamma_{n-1} a_{n-1, k}, & n \geq 2, k<n \\ \left(1-\gamma_{n-1}\right), & n \geq 2, k=n .\end{cases}
$$


Then the Mann-Toeplitz iterative method (1.6) becomes

$$
v_{n+1}=\gamma_{n} v_{n}+\left(1-\gamma_{n}\right) f_{\mu_{n}} \sum_{k=0}^{n-1} b_{n, k+1} T^{k} v_{n} .
$$

The use of this matrix of regularization will be that it permits us to improve the main theorem of Dotson [24], obtaining strong convergence instead of weak convergence. Indeed, we will prove the following.

Theorem 1.4 Let $f: H \rightarrow H$ be a $\rho$-contraction and let $f_{\mu_{n}}$ be the averaged mapping $f_{\mu_{n}}=$ $\mu_{n} f+\left(1-\mu_{n}\right) I$. Let $T$ be a nonspreading mapping. Let $\left(b_{n, k}\right)_{n, k \in \mathbb{N}} \in \mathfrak{T}$ be a triangularToeplitz matrix.

Let us choose $\mu<\frac{(1-\rho)}{(1+\rho)^{2}}$ and $\left(\mu_{n}\right)_{n \in \mathbb{N}} \subset(0, \mu)$ such that

(h4) $\lim _{n \rightarrow \infty} \mu_{n}=0, \sum_{n \in \mathbb{N}} \mu_{n}=\infty$.

Let us take $\left(\tilde{a}_{n, k}\right)_{n, k \in \mathbb{N}} \in \mathfrak{M}$ defined by a sequence $\left(\gamma_{n}\right)_{n \in \mathbb{N}} \subset[0, \alpha] \subset[0,1)$ (see (1.8)) such that

(h6) $\lim _{n \rightarrow \infty} \gamma_{n}=0$.

Then the sequence generated by $v_{1} \in H$ and by the iteration (1.9) strongly converges to $x^{*} \in$ $\operatorname{Fix}(T)$, that is, the unique solution of the variational inequality

$$
\left\langle(I-f) x^{*}, y-x^{*}\right\rangle \geq 0, \quad \forall y \in \operatorname{Fix}(T) .
$$

\section{Proofs}

First we prove Lemma 1.1, which is a fundamental tool to prove the convergence of our methods.

Proof of Lemma 1.1 Note that since $\left(b_{n, k}\right)_{n, k \in N}$ is nonincreasing, $0 \leq b_{n, n} \leq b_{n, k}$, for any $k \leq n$, so (h3) implies that $b_{n, n} \rightarrow 0$, as $n \rightarrow \infty$.

Moreover, following [25], one easily obtains

$$
\begin{gathered}
\left\|T^{k+1} x-T y\right\|^{2} \leq \\
+2 T^{k} x-T y\left\|^{2}+\right\| T y-y \|^{2}+2\left\langle T^{k} x-T y, T y-y\right\rangle \\
+2\left\langle T^{k} x-T^{k+1} x, y-T y\right|, \quad \forall k \in \mathbb{N} .
\end{gathered}
$$

Therefore the following inequality holds:

$$
\begin{aligned}
\left\|T^{k+1} x_{n}-T y\right\|^{2}-\left\|T^{k} x_{n}-T y\right\|^{2} \leq & \|T y-y\|^{2}+2\left\langle T^{k} x_{n}-T y, T y-y\right\rangle \\
& +2\left\langle T^{k} x_{n}-T^{k+1} x_{n}, y-T y\right\rangle .
\end{aligned}
$$

Firstly we work on the right side of the previous equation. Multiplying by $b_{n, k+1}$ and summing up over $k$ from 0 to $(n-1)$, by (h2) and (1.3) we obtain

$$
\begin{aligned}
\sum_{k=0}^{n-1} b_{n, k+1}\|T y-y\|^{2}+2 \sum_{k=0}^{n-1} b_{n, k+1}\left\langle T^{k} x_{n}-T y, T y-y\right\rangle & \\
& +2 \sum_{k=0}^{n-1} b_{n, k+1}\left\langle T^{k} x_{n}-T^{k+1} x_{n}, y-T y\right\rangle \\
= & \|T y-y\|^{2}+2\left\langle y_{n}-T y, T y-y\right\rangle+2\left\langle y_{n}-\sum_{k=0}^{n-1} b_{n, k+1} T^{k+1} x_{n}, y-T y\right\rangle .
\end{aligned}
$$


We observe that

$$
\begin{aligned}
y_{n}-\sum_{k=0}^{n-1} b_{n, k+1} T^{k+1} x_{n} & =\sum_{k=0}^{n-1} b_{n, k+1} T^{k} x_{n}-\sum_{k=0}^{n-1} b_{n, k+1} T^{k+1} x_{n} \\
& =b_{n, 1} x_{n}+\sum_{k=1}^{n-1}\left(b_{n, k+1}-b_{n, k}\right) T^{k} x_{n}+b_{n, n} T^{n} x_{n}
\end{aligned}
$$

then, by the boundedness of $\left(x_{n}\right)_{n \in \mathbb{N}}$ and by the quasi-nonexpansivity of $T$, there exists $M=M(y)$ such that, by (h1), (2.2) becomes

$$
\begin{aligned}
\| T y & -y \|^{2}+2\left\langle y_{n}-T y, T y-y\right\rangle+2\left\langle y_{n}-\sum_{k=0}^{n-1} b_{n, k+1} T^{k+1} x_{n}, y-T y\right\rangle \\
= & \|T y-y\|^{2}+2\left\langle y_{n}-T y, T y-y\right\rangle \\
& +2\left[b_{n, 1}\left\langle x_{n}, y-T y\right\rangle+\sum_{k=1}^{n-1}\left(b_{n, k+1}-b_{n, k}\right)\left\langle T^{k} x_{n}, y-T y\right\rangle+b_{n, n}\left\langle T^{n} x_{n}, y-T y\right\rangle\right] \\
\leq & \|T y-y\|^{2}+2\left\langle y_{n}-T y, T y-y\right\rangle+2 M\left[b_{n, 1}+\sum_{k=1}^{n-1}\left(b_{n, k+1}-b_{n, k}\right)+b_{n, n}\right] \\
= & \|T y-y\|^{2}+2\left\langle y_{n}-T y, T y-y\right\rangle+4 M b_{n, n} .
\end{aligned}
$$

In a similar manner, for the left side of (2.1), multiplying by $b_{n, k+1}$, summing over $k$ from 0 to $(n-1)$ and using (h1), we have

$$
\begin{aligned}
& \sum_{k=0}^{n-1} b_{n, k+1}\left\|T^{k+1} x_{n}-T y\right\|^{2}-\sum_{k=0}^{n-1} b_{n, k+1}\left\|T^{k} x_{n}-T y\right\|^{2} \\
& \quad=b_{n, n}\left\|T^{n} x_{n}-T y\right\|^{2}-b_{n, 1}\left\|x_{n}-T y\right\|^{2}+\sum_{k=1}^{n-1}\left(b_{n, k}-b_{n, k+1}\right)\left\|T^{k} x_{n}-T y\right\|^{2} \\
& \quad \geq b_{n, n}\left\|T^{n} x_{n}-T y\right\|^{2}-b_{n, 1}\left\|x_{n}-T y\right\|^{2} .
\end{aligned}
$$

Therefore we have the following chain:

$$
\begin{aligned}
b_{n, n} \| & T^{n} x_{n}-T y\left\|^{2}-b_{n, 1}\right\| x_{n}-T y \|^{2} \\
\leq & \sum_{k=0}^{n-1} b_{n, k+1}\left\|T^{k+1} x_{n}-T y\right\|^{2}-\sum_{k=0}^{n-1} b_{n, k+1}\left\|T^{k} x_{n}-T y\right\|^{2} \\
\leq & \|T y-y\|^{2}+2\left\langle y_{n}-T y, T y-y\right\rangle \\
& +2\left\langle y_{n}-\sum_{k=0}^{n-1} b_{n, k+1} T^{k+1} x_{n}, y-T y\right\rangle+b_{n, 1}\left\|x_{n}-T y\right\|^{2} \\
\leq & \|T y-y\|^{2}+2\left\langle y_{n}-T y, T y-y\right\rangle+4 M b_{n, 1}
\end{aligned}
$$


and so, by the positivity of $b_{n, k}$, it follows that

$$
b_{n, n}\left\|T^{n} x_{n}-T y\right\|^{2} \leq\|T y-y\|^{2}+2\left\langle y_{n}-T y, T y-y\right\rangle+4 M b_{n, n}+2 M b_{n, 1} .
$$

Now, if $w \in \omega_{w}\left(y_{n}\right)$, by $b_{n, n} \rightarrow 0$ as $n \rightarrow+\infty$, we have the result that, for every $y \in H$,

$$
0 \leq\|T y-y\|^{2}+2\langle w-T y, T y-y\rangle
$$

so, choosing $y=w$

$$
\|T w-w\|^{2} \leq 0
$$

i.e. we have the thesis.

To obtain our results we also need of the following (well-known) lemmas; the first is proved in [26], the second is the discrete Jensen inequality.

Lemma 2.1 [26] Assume $\left(a_{n}\right)_{n \in \mathbb{N}}$ is a sequence of nonnegative numbers for which

$$
a_{n+1} \leq\left(1-s_{n}\right) a_{n}+\delta_{n}, \quad n \geq 0,
$$

where $\left(s_{n}\right)_{n \in \mathbb{N}}$ is a sequence in $(0,1)$ and $\left(\delta_{n}\right)_{n \in \mathbb{N}}$ is a sequence in $\mathbb{R}$ such that

(1) $\lim _{n \rightarrow \infty} s_{n}=0$ and $\sum_{n=1}^{\infty} s_{n}=\infty$;

(2) $\lim \sup _{n \rightarrow \infty} \frac{\delta_{n}}{s_{n}} \leq 0$ or $\sum_{n=1}^{\infty}\left|\delta_{n}\right|<\infty$.

Then $\lim _{n \rightarrow \infty} a_{n}=0$.

Lemma 2.2 Let $\psi: H \rightarrow \mathbb{R}$ be a convex function. Let $\left\{x_{1}, \ldots, x_{n}\right\} \subset H$ and let positive weights $\left\{a_{1}, \ldots, a_{n}\right\} \subset \mathbb{R}$, we have the result that

$$
\psi\left(\frac{\sum_{i=1}^{n} a_{i} x_{i}}{\sum_{i=1}^{n} a_{i}}\right) \leq \frac{\sum_{i=1}^{n} a_{i} \psi\left(x_{i}\right)}{\sum_{i=1}^{n} a_{i}}
$$

Proof of Theorem 1.2 It is well known that (see [27])

$$
\left\|f_{\mu_{n}} x-f_{\mu_{n}} y\right\| \leq\left(1-\mu_{n} \tau\right)\|x-y\|,
$$

where $\tau:=1-\rho$.

Moreover, we will denote $y_{n}=\sum_{k=0}^{n-1} b_{n, k+1} T^{k} v_{n}$. Since $T$ is quasi-nonexpansive and (h2) holds, for every $w \in \operatorname{Fix}(T)$, as a result one finds that

$$
\left\|y_{n}-w\right\| \leq \sum_{k=0}^{n-1} b_{n, k+1}\left\|T^{k} v_{n}-T^{k} w\right\| \leq \sum_{k=0}^{n-1} b_{n, k+1}\left\|v_{n}-w\right\|=\left\|v_{n}-w\right\| .
$$

Set

$$
r:=\max \left\{\left\|x_{1}-w\right\|, \frac{\|(I-f) w\|}{\tau}\right\}
$$


we prove, by induction on $n$, that $\left(x_{n}\right)_{n \in \mathbb{N}}$ and $\left(v_{n}\right)_{n \in \mathbb{N}} \subset B(w, r)$. Let us observe the following:

$$
\begin{aligned}
\left\|x_{n+1}-w\right\| & \leq\left\|f_{\mu_{n}} y_{n}-w\right\| \\
& \leq\left\|f_{\mu_{n}} y_{n}-f_{\mu_{n}} w\right\|+\left\|f_{\mu_{n}} w-w\right\| \\
& \leq\left(1-\mu_{n} \tau\right)\left\|y_{n}-w\right\|+\left\|f_{\mu_{n}} w-w\right\| \\
& \leq\left(1-\mu_{n} \tau\right)\left\|v_{n}-w\right\|+\mu_{n}\|(I-f) w\| \\
& =\left(1-\mu_{n} \tau\right)\left\|\sum_{k=1}^{n} a_{n, k}\left(x_{k}-w\right)\right\|+\tau \mu_{n} \frac{\|(I-f) w\|}{\tau} .
\end{aligned}
$$

Since $x_{1}=v_{1} \in B(w, r)$, we immediately have $x_{2} \in B(w, r)$ and then, by (M2), $v_{2} \in B(w, r)$.

Let us suppose that $x_{i} \in B(w, r)$, for every $i=1, \ldots, n$. Then, by (M2), $v_{n} \in B(w, r)$. Therefore by the previous results we have

$$
\left\|x_{n+1}-w\right\| \leq\left(1-\mu_{n} \tau\right) r+\tau \mu_{n} r=r
$$

and $\left\|v_{n+1}-w\right\| \leq r$.

The next step is to prove that the weak cluster points of $\left(x_{n}\right)_{n \in \mathbb{N}}$ are fixed points of $T$. First we note that

$$
\begin{aligned}
\left\|x_{n+1}-y_{n}\right\| & =\left\|f_{\mu_{n}} y_{n}-y_{n}\right\| \\
& =\left\|\mu_{n}(I-f) y_{n}\right\| \\
& \leq \mu_{n}\left\|(I-f) y_{n}\right\|,
\end{aligned}
$$

so, since $\left(\mu_{n}\right)_{n \in \mathbb{N}}$ is a null sequence (see (h4)), $\omega_{w}\left(x_{n}\right)=\omega_{w}\left(y_{n}\right)$. By the boundedness of $\left(x_{n}\right)_{n \in \mathbb{N}}$, Lemma 1.1 ensures that the weak limits of the sequence $\left(y_{n}\right)_{n \in \mathbb{N}}$ are contained in the set fixed points of $T$. The previous inequality shows that $\omega_{w}\left(x_{n}\right) \subset \operatorname{Fix}(T)$.

Finally, if $x^{*} \in \operatorname{Fix}(T)$ is the unique solution of a (VIP), as a result one finds that

$$
\begin{aligned}
\left\|x_{n+1}-x^{*}\right\|^{2}= & \left\|f_{\mu_{n}} y_{n}-x^{*} \pm\left(1-f_{\mu_{n}} x^{*}\right)\right\|^{2} \\
= & \left\|\left(f_{\mu_{n}} y_{n}-f_{\mu_{n}} x^{*}\right)-\mu_{n}(I-f) x^{*}\right\|^{2} \\
\leq & \left\|\left(f_{\mu_{n}} y_{n}-f_{\mu_{n}} x^{*}\right)\right\|^{2} \\
& -2 \mu_{n}\left((I-f) x^{*}, x_{n+1}-x^{*}\right\rangle \\
\leq & \left(1-\mu_{n} \tau\right)^{2}\left\|v_{n}-x^{*}\right\|^{2} \\
& +2 \mu_{n}\left(-(I-f) x^{*}, x_{n+1}-x^{*}\right\rangle \\
= & \left(1-\mu_{n} \tau\right)^{2}\left\|\sum_{k=1}^{n} a_{n, k}\left(x_{k}-x^{*}\right)\right\|^{2} \\
& +2 \mu_{n}\left(-(I-f) x^{*}, x_{n+1}-x^{*}\right\rangle \\
\leq & \left(1-\mu_{n} \tau\right)^{2} \sum_{k=1}^{n} a_{n, k}\left\|x_{k}-x^{*}\right\|^{2}
\end{aligned}
$$




$$
\begin{aligned}
& +2 \mu_{n}\left\langle-(I-f) x^{*}, x_{n+1}-x^{*}\right\rangle \\
= & \left(1-\mu_{n} \tau\right)^{2} a_{n, n}\left\|x_{n}-x^{*}\right\|^{2} \\
& +\left(1-\mu_{n} \tau\right)^{2} \sum_{k=1}^{n-1} a_{n, k}\left\|x_{k}-x^{*}\right\|^{2}+2 \mu_{n}\left(-(I-f) x^{*}, x_{n+1}-x^{*}\right\rangle \\
\leq & \left(1-\mu_{n} \tau\right)^{2}\left\|x_{n}-x^{*}\right\|^{2} \\
& +r^{2} \sum_{k=1}^{n-1} a_{n, k}+2 \mu_{n}\left(-(I-f) x^{*}, x_{n+1}-x^{*}\right\rangle \\
= & {\left[1+\left(\mu_{n}^{2} \tau^{2}-2 \mu_{n} \tau\right)\right]\left\|x_{n}-x^{*}\right\|^{2} } \\
& +\left(1-a_{n, n}\right) r^{2}+2 \mu_{n}\left(-(I-f) x^{*}, x_{n+1}-x^{*}\right\rangle \\
= & {\left[1-\mu_{n} \tau\left(2-\mu_{n} \tau\right)\right]\left\|x_{n}-x^{*}\right\|^{2} } \\
& +\left(1-a_{n, n}\right) r^{2}+2 \mu_{n}\left(-(I-f) x^{*}, x_{n+1}-x^{*}\right\rangle .
\end{aligned}
$$

Since $\left(x_{n}\right)_{n \in \mathbb{N}}$ is bounded, there exists a subsequence $x_{n_{k}} \rightarrow z_{0}$ such that

$$
\underset{n}{\limsup }\left\langle-(I-f) x^{*}, x_{n+1}-x^{*}\right\rangle=\lim _{k}\left\langle-(I-f) x^{*}, x_{n_{k}+1}-x^{*}\right\rangle=\left\langle-(I-f) x^{*}, z_{0}-x^{*}\right\rangle
$$

Since every weak cluster point of $\left(x_{n}\right)_{n \in \mathbb{N}}$ is in $\operatorname{Fix}(T)$,

$$
\limsup _{n}\left\langle-(I-f) x^{*}, x_{n+1}-x^{*}\right\rangle=\left\langle-(I-f) x^{*}, z_{0}-x^{*}\right\rangle \leq 0 .
$$

By (h4), (h5), (h6), and by using Lemma 2.1 we obtain $x_{n} \rightarrow x^{*}$.

Proof of Theorem 1.4 Also here we will denote $y_{n}=\sum_{k=0}^{n-1} b_{n, k+1} T^{k} v_{n}$. Since $T$ is quasinonexpansive and (h2) holds, for all $w \in \operatorname{Fix}(T)$, as a result one finds that

$$
\left\|y_{n}-w\right\| \leq \sum_{k=0}^{n-1} b_{n, k+1}\left\|T^{k} v_{n}-T^{k} w\right\| \leq \sum_{k=0}^{n-1} b_{n, k+1}\left\|v_{n}-w\right\|=\left\|v_{n}-w\right\| .
$$

If

$$
r:=\max \left\{\left\|v_{1}-w\right\|, \frac{\|(I-f) w\|}{\tau}\right\}
$$

we prove, by induction on $n$, that $\left(v_{n}\right)_{n \in \mathbb{N}} \subset B(w, r)$. Set $\tau=1-\rho$, let us observe the following:

$$
\begin{aligned}
\left\|v_{n+1}-w\right\| & \leq \gamma_{n}\left\|v_{n}-w\right\|+\left(1-\gamma_{n}\right)\left\|f_{\mu_{n}} y_{n}-w\right\| \\
& \leq \gamma_{n}\left\|v_{n}-w\right\|+\left(1-\gamma_{n}\right)\left\|f_{\mu_{n}} y_{n}-f_{\mu_{n}} w\right\|+\left(1-\gamma_{n}\right)\left\|f_{\mu_{n}} w-w\right\| \\
& \leq \gamma_{n}\left\|v_{n}-w\right\|+\left(1-\gamma_{n}\right)\left(1-\mu_{n} \tau\right)\left\|y_{n}-w\right\|+\left(1-\gamma_{n}\right)\left\|f_{\mu_{n}} w-w\right\| \\
& \leq \gamma_{n}\left\|v_{n}-w\right\|+\left(1-\gamma_{n}\right)\left(1-\mu_{n} \tau\right)\left\|v_{n}-w\right\|+\left(1-\gamma_{n}\right) \mu_{n}\|(I-f) w\| \\
& =\gamma_{n}\left\|v_{n}-w\right\|+\left(1-\gamma_{n}\right)\left(1-\mu_{n} \tau\right)\left\|\left(v_{n}-w\right)\right\|+\tau \mu_{n}\left(1-\gamma_{n}\right) \frac{\|(I-f) w\|}{\tau} .
\end{aligned}
$$


Now, $v_{1} \in B(w, r)$. For induction, let us suppose that $v_{i} \in B(w, r)$, for every $i=1, \ldots, n$. Therefore by the previous results we have

$$
\left\|v_{n+1}-w\right\| \leq \gamma_{n} r+\left(1-\gamma_{n}\right)\left(1-\mu_{n} \tau\right) r+\tau \mu_{n}\left(1-\gamma_{n}\right) r=r
$$

and $\left\|v_{n+1}-w\right\| \leq r$.

Now we want to prove that the weak cluster points of $\left(v_{n}\right)_{n \in \mathbb{N}}$ are fixed points of $T$. First we note that

$$
\begin{aligned}
\left\|v_{n+1}-y_{n}\right\| & =\left\|\gamma_{n} v_{n}+\left(1-\gamma_{n}\right) f_{\mu_{n}} y_{n}-y_{n}\right\| \\
& =\left\|\gamma_{n}\left(v_{n}-y_{n}\right)+\left(1-\gamma_{n}\right) \mu_{n}(I-f) y_{n}\right\| \\
& \leq \gamma_{n}\left\|v_{n}-y_{n}\right\|+\mu_{n}\left\|(I-f) y_{n}\right\|,
\end{aligned}
$$

thus, since $\left(\mu_{n}\right)_{n \in \mathbb{N}},\left(\gamma_{n}\right)_{n \in \mathbb{N}}$ are null sequences (see (h4) and (h6)), $\omega_{w}\left(v_{n}\right)=\omega_{w}\left(y_{n}\right)$. By the boundedness of $\left(v_{n}\right)_{n \in \mathbb{N}}$, Lemma 1.1 guarantees that the weak limits of the sequence $\left(y_{n}\right)_{n \in \mathbb{N}}$ are contained in the set of fixed points of $T$. The previous inequality shows that $\omega_{w}\left(v_{n}\right) \subset \operatorname{Fix}(T)$.

Lastly, if $x^{*} \in \operatorname{Fix}(T)$ is the unique solution of a (VIP), as a result one finds that

$$
\begin{aligned}
\left\|v_{n+1}-x^{*}\right\|^{2}= & \left\|\gamma_{n} v_{n}+\left(1-\gamma_{n}\right) f_{\mu_{n}} y_{n}-x^{*} \pm\left(1-\gamma_{n}\right) f_{\mu_{n}} x^{*}\right\|^{2} \\
= & \left\|\gamma_{n}\left(v_{n}-x^{*}\right)+\left(1-\gamma_{n}\right)\left(f_{\mu_{n}} y_{n}-f_{\mu_{n}} x^{*}\right)-\left(1-\gamma_{n}\right) \mu_{n}(I-f) x^{*}\right\|^{2} \\
\leq & \left\|\gamma_{n}\left(v_{n}-x^{*}\right)+\left(1-\gamma_{n}\right)\left(f_{\mu_{n}} y_{n}-f_{\mu_{n}} x^{*}\right)\right\|^{2} \\
& -2\left(1-\gamma_{n}\right) \mu_{n}\left\langle(I-f) x^{*}, x_{n+1}-x^{*}\right\rangle \\
\leq & \gamma_{n}\left\|v_{n}-x^{*}\right\|^{2}+\left(1-\gamma_{n}\right)\left(1-\mu_{n} \tau\right)^{2}\left\|v_{n}-x^{*}\right\|^{2} \\
& +2\left(1-\gamma_{n}\right) \mu_{n}\left(-(I-f) x^{*}, v_{n+1}-x^{*}\right\rangle \\
= & {\left[1+\left(1-\gamma_{n}\right)\left(\mu_{n}^{2} \tau^{2}-2 \mu_{n} \tau\right)\right]\left\|v_{n}-x^{*}\right\|^{2} } \\
& +2\left(1-\gamma_{n}\right) \mu_{n}\left(-(I-f) x^{*}, v_{n+1}-x^{*}\right\rangle \\
= & {\left[1-\left(1-\gamma_{n}\right) \mu_{n} \tau\left(2-\mu_{n} \tau\right)\right]\left\|v_{n}-x^{*}\right\|^{2} } \\
& +2\left(1-\gamma_{n}\right) \mu_{n}\left(-(I-f) x^{*}, x_{n+1}-x^{*}\right\rangle .
\end{aligned}
$$

Since $\left(v_{n}\right)_{n \in \mathbb{N}}$ is bounded, there exists a subsequence $v_{n_{k}} \rightarrow z_{0}$ such that

$$
\limsup _{n}\left\langle-(I-f) x^{*}, v_{n+1}-x^{*}\right\rangle=\lim _{k}\left\langle-(I-f) x^{*}, v_{n_{k}+1}-x^{*}\right\rangle=\left\langle-(I-f) x^{*}, z_{0}-x^{*}\right\rangle .
$$

Since every weak cluster point of $\left(v_{n}\right)_{n \in \mathbb{N}}$ is in $\operatorname{Fix}(T)$,

$$
\limsup _{n}\left\langle-(I-f) x^{*}, v_{n+1}-x^{*}\right\rangle=\left\langle-(I-f) x^{*}, z_{0}-x^{*}\right\rangle \leq 0 .
$$

By (h4) and (h6) and by using Lemma 2.1 we obtain $v_{n} \rightarrow x^{*}$. 
Remark 2.3 If $\left(b_{n, k}\right)_{n, k \in \mathbb{N}}$ is generated recursively by iteration (1.8) and by a sequence $\left(\eta_{n}\right)_{n \in \mathbb{N}}$ such that

$$
\eta_{0}=1, \quad \eta_{n+1} \leq \frac{\eta_{n}}{1+\eta_{n}}
$$

(e.g. $\eta_{n}=\frac{1}{(n+1)^{\eta}}$ with $\left.\eta \geq 1\right)$ then $\left(b_{n, k}\right)_{n, k \in \mathbb{N}}$ belongs to $\mathfrak{T}$.

\section{Competing interests}

The authors declare that they have no competing interests.

\section{Authors' contributions}

All authors contributed equally and significantly in writing this article. All authors read and approved the final manuscript.

\section{Acknowledgements}

This article was funded by the Deanship of Scientific Research (DSR), King Abdulaziz University, Jeddah. Therefore, the first author acknowledges with thanks DSR, KAU, for financial support.

\section{Received: 7 January 2015 Accepted: 5 May 2015 Published online: 20 May 2015}

\section{References}

1. Moudafi, A: Viscosity approximation methods for fixed-points problems. J. Math. Anal. Appl. 241, 46-55 (2000)

2. Xu, HK: Viscosity approximation methods for nonexpansive mappings. J. Math. Anal. Appl. 298, 279-291 (2004)

3. Marino, G, Xu, HK: A general iterative method for nonexpansive mappings in Hilbert spaces. J. Math. Anal. Appl. 318, 43-52 (2006)

4. Kohsaka, F, Takahashi, W: Fixed point theorems for a class of nonlinear mappings related to maximal monotone operators in Banach spaces. Arch. Math. (Basel) 91(2), 166-177 (2008)

5. Kohsaka, F, Takahashi, W: Existence and approximation of fixed points of firmly nonexpansive-type mappings in Banach spaces. SIAM J. Optim. 19(2), 824-835 (2008)

6. lemoto, S, Takahashi, W: Approximating common fixed points of nonexpansive mappings and nonspreading mappings in a Hilbert space. Nonlinear Anal. 71(12), 2082-2089 (2009)

7. Maingé, PE: The viscosity approximation process for quasi-nonexpansive mappings in Hilbert spaces. Comput. Math. Appl. 59(1), 74-79 (2010)

8. Hussain, N, Takahashi, W: Weak and strong convergence theorems for semigroups of mappings without continuity in Hilbert spaces. J. Nonlinear Convex Anal. 14(4), 769-783 (2013)

9. Cooke, RS: Infinite Matrices and Sequence Spaces. Macmillan \& Co., London (1950)

10. Mann, WR: Mean value methods in iteration. Proc. Am. Math. Soc. 4, 506-510 (1953)

11. Hussain, N, Marino, G, Abdou Afrah, AN: On Mann's method with viscosity for nonexpansive and nonspreading mappings in Hilbert spaces. Abstr. Appl. Anal. 2014, Article ID 152530 (2014)

12. Baillon, JB: Un théorème de type ergodique pour les contractions non linéairs dans un espace de Hilbert. C. R. Acad. Sci. Paris Sér. A-B 280, 1511-1541 (1975)

13. Shimizu, T, Takahashi, W: Strong convergence theorem to common fixed points of families of nonexpansive mappings. J. Math. Anal. Appl. 211, 71-83 (1997)

14. Shioji, N, Takahashi, W: A strong convergence theorem for asymptotically nonexpansive mappings in Banach spaces. Arch. Math. 72, 354-359 (1999)

15. Song, Y, Chen, R: Viscosity approximative methods to Cesáro means for non-expansive mappings. Appl. Math. Comput. 186(2), 1120-1128 (2007)

16. Matsushita, S, Kuroiwa, D: Strong convergence of averaging iterations of nonexpansive nonself-mappings. J. Math. Anal. Appl. 294, 206-214 (2004)

17. Su, Y, Li, S: Strong convergence theorems on two iterative method for non-expansive mappings. Appl. Math. Comput. 181, 332-341 (2006)

18. Wangkeeree, R: Viscosity approximative methods to Cesáro means iterations for nonexpansive nonself-mappings in Banach spaces. Appl. Math. Comput. 201, 239-249 (2008)

19. Aoyama, K, Takahashi, W: Weak convergence theorems by Cesáro means for a nonexpansive mappings and an equilibrium problem. Pac. J. Optim. 3(3), 501-509 (2007)

20. liduka, H, Takahashi, W: Weak convergence theorems by Cesáro means for nonexpansive mappings and inverse-strongly-monotone mappings. J. Nonlinear Convex Anal. 7, 105-113 (2006)

21. Jitpeera, T, Katchang, P, Kumam, P: A viscosity of Cesáro mean approximation methods for a mixed equilibrium variational inequalities, and fixed point problems. Fixed Point Theory Appl. 2011, Article ID 945051 (2011)

22. Song, Y: Iterative convergence to Cesáro means for continuous pseudocontractive mappings. Nonlinear Anal. 71 2792-2800 (2009)

23. Song, Y: Mann iteration of Cesáro means for asymptotically non-expansive mappings. Nonlinear Anal. 72, 176-182 (2010)

24. Dotson, WG, Jr: On the Mann iterative process. Trans. Am. Math. Soc. 149, 65-73 (1970)

25. Osilike, MO, Isiogugu, FO: Weak and strong convergence theorems for nonspreading-type mappings in Hilbert spaces. Nonlinear Anal. 74(5), 1814-1822 (2011)

26. Xu, HK: An iterative approach to quadratic optimization. J. Optim. Theory Appl. 116(3), 659-678 (2003)

27. $\mathrm{Xu}, \mathrm{HK}, \mathrm{Kim}, \mathrm{TH}$ : Convergence of hybrid steepest-descent methods for variational inequalities. J. Optim. Theory Appl. 119(1), 185-201 (2003) 